Supplement of Hydrol. Earth Syst. Sci., 23, 4527-4539, 2019

https://doi.org/10.5194/hess-23-4527-2019-supplement

(C) Author(s) 2019. This work is distributed under

the Creative Commons Attribution 4.0 License.

(c) (1)

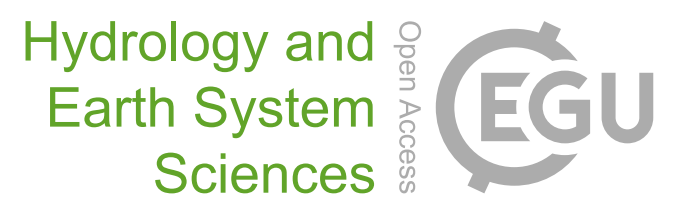

Supplement of

\title{
Groundwater-glacier meltwater interaction in proglacial aquifers
}

Brighid É. Ó Dochartaigh et al.

Correspondence to: Alan M. MacDonald (amm@bgs.ac.uk)

The copyright of individual parts of the supplement might differ from the CC BY 4.0 License. 
Table S1 Summary of sandur piezometer locations, construction and test pumping data (For more information see Ó Dochartaigh et al., 2012)

\begin{tabular}{|c|c|c|c|c|c|c|c|c|}
\hline ID & Easting & Northing & $\begin{array}{l}\text { screen } \\
\text { depth }(m)\end{array}$ & $\begin{array}{l}\text { Test } \\
\text { length } \\
\text { (min) } \\
\end{array}$ & $\begin{array}{l}\text { Test } \\
\text { yield } \\
\left(\mathbf{m}^{\mathbf{3}} \mathbf{d}^{-1}\right) \\
\end{array}$ & $\begin{array}{l}\text { Maximum } \\
\text { drawdown } \\
(\mathrm{m})\end{array}$ & $\mathbf{T}\left(\mathbf{m}^{2} \mathbf{d}^{-1}\right)$ & Comments \\
\hline U1 & 63.959056 & -16.836639 & $10.9-13.9$ & 300 & 95.04 & 3.81 & 100 & \\
\hline U2 & 63.959417 & -16.837833 & $\begin{array}{r}9.2-10.2 \\
12.2-13.2\end{array}$ & 225 & 138.24 & 0.7 & 600 & \\
\hline M1 & 63.954194 & -16.848306 & $11.4-14.4$ & 300 & 112.32 & 3.46 & 200 & \\
\hline $\mathrm{M} 2$ & 63.955000 & -16.848583 & $12.2-14.2$ & 225 & 95.04 & 3.7 & 150 & \\
\hline M3 & 63.958028 & -16.850083 & $8.25-14.25$ & 230 & 120.96 & 0.85 & 600 & \\
\hline $\mathrm{L} 1$ & 63.942472 & -16.857083 & $5.5-11.5$ & 360 & 43.2 & 7.02 & 80 & $\begin{array}{l}\text { Test results } \\
\text { affected by } \\
\text { poor borehole } \\
\text { construction }\end{array}$ \\
\hline L2 & 63.943833 & -16.858222 & $4.8-7.8$ & 244 & 157.248 & 0.38 & 2500 & \\
\hline L3 & 63.946694 & -16.860500 & $4.9-7.9$ & 215 & 150.336 & 0.42 & 2000 & \\
\hline
\end{tabular}

Ó Dochartaigh, B.É., MacDonald, A.M., Wilson, P.R., Bonsor, H.: Groundwater investigations at Virkisjokull, Iceland : data report 2012. Nottingham, UK, British Geological Survey, 48pp. (OR/12/088), 2012. 
Table S2 Summary of saturated hydraulic conductivity measurements for sandur aquifer from direct infiltration tests to $0.15 \mathrm{~m}$ depth.

\begin{tabular}{|c|c|c|c|c|c|c|}
\hline Test ID & Northing & Easting & $\begin{array}{l}\text { Hole } \\
\text { depth } \\
(\mathrm{mm}) \\
\end{array}$ & $\begin{array}{l}\text { Hole } \\
\text { diameter } \\
(\mathbf{m m})\end{array}$ & $\begin{array}{l}5 \mathrm{~cm} \text { head } \\
\text { infiltration rate } \\
\left(\mathrm{cm} \mathrm{s}^{-1}\right)\end{array}$ & $\begin{array}{l}\text { Kfs (m } \\
\left.d^{-1}\right)\end{array}$ \\
\hline Site 1 & 63.95299 & -16.84898 & 120 & 65 & 0.12 & 14.83 \\
\hline Site 2 & 63.95345 & -16.84853 & 140 & 70 & 0.85 & 96.62 \\
\hline Site 3 & 63.95390 & -16.84837 & 100 & 60 & 0.86 & 109.4 \\
\hline Site 4 & 63.95451 & -16.84773 & 140 & 65 & 1.07 & 124.4 \\
\hline Site 5 & 63.95491 & -16.84679 & 120 & 60 & 0.3 & 36.58 \\
\hline Site 6 & 63.95500 & -16.84690 & 140 & 60 & 0.5 & 59.62 \\
\hline Site 7 & 63.95700 & -16.84710 & 140 & 50 & 0.04 & 5.47 \\
\hline Site 8 & 63.95511 & -16.84616 & 100 & 120 & 1.2 & 72.86 \\
\hline Site 9 & 63.95634 & -16.84602 & 120 & 60 & 0.69 & 77.47 \\
\hline Site 10 & 63.95759 & -16.84697 & 120 & 50 & 0.74 & 94.46 \\
\hline Site 11 & 63.95760 & -16.84500 & 150 & 100 & 0.45 & 30.96 \\
\hline $28 / 8(1)$ & 63.94364 & -16.85847 & 110 & 80 & 0.21 & 22.03 \\
\hline $28 / 8(2)$ & 63.94381 & -16.85700 & 140 & 80 & 0.45 & 47.23 \\
\hline $30 / 8(1)$ & 63.94114 & -16.85653 & 150 & 60 & 0.08 & 10.96 \\
\hline $30 / 8(2)$ & 63.93800 & -16.85750 & 150 & 100 & 0.7 & 59.20 \\
\hline $30 / 8(3)$ & 63.94100 & -16.85781 & 150 & 70 & 0.12 & 14.27 \\
\hline $30 / 8(4)$ & 63.94092 & -16.85789 & 150 & 100 & 0.25 & 21.18 \\
\hline $30 / 8(5)$ & 63.94272 & -16.85789 & 150 & 70 & 0.42 & 49.84 \\
\hline $7 / 9(1)$ & 63.94261 & -16.85475 & 110 & 60 & 0.8 & 108.7 \\
\hline $7 / 9(2)$ & 63.94275 & -16.85450 & 120 & 70 & 1.07 & 126.9 \\
\hline
\end{tabular}


Table S3 Summary of hydraulic conductivity measurements from particle size analysis of sandur aquifer sediment samples to $0.5 \mathrm{~m}$ depth. Hydraulic conductivity calculated using MacDonald et al. (2012).

\begin{tabular}{|c|c|c|c|c|c|c|}
\hline Test ID & Northing & Easting & $\begin{array}{l}\text { Hole depth } \\
\text { (m) }\end{array}$ & $\begin{array}{l}\text { Hole size } \\
(\mathrm{mm})\end{array}$ & d10 & $\mathrm{K} \mathrm{m} / \mathrm{d}$ \\
\hline A1.1.1 & 63.95911 & -16.83670 & 0.46 & $1000 * 500$ & 0.67 & 38.25 \\
\hline A1.1.2 & 63.95911 & -16.83675 & 0.46 & $1000 * 500$ & 0.19 & 14.13 \\
\hline A1.2.1 & 63.95914 & -16.83667 & 0.43 & $800 * 500$ & 0.27 & 18.65 \\
\hline A1.2.2 & 63.95913 & -16.83667 & 0.43 & $800 * 500$ & 0.19 & 14.13 \\
\hline A2.1.1 & 63.95936 & -16.83811 & 0.45 & $850 * 500$ & 0.48 & 29.39 \\
\hline A 2.1 .2 & 63.95936 & -16.83811 & 0.45 & $850 * 500$ & 0.4 & 25.45 \\
\hline A2.2.1 & 63.95936 & -16.83819 & 0.45 & $700 * 400$ & 0.4 & 25.45 \\
\hline A 2.2 .2 & 63.95936 & -16.83819 & 0.45 & $700 * 400$ & 0.41 & 25.95 \\
\hline B1.1.2 & 63.95614 & -16.84236 & 0.46 & $450 * 560$ & 0.7 & 39.59 \\
\hline B1.2.1 & 63.95619 & -16.84228 & 0.46 & $1000 * 650$ & 1.8 & 83.50 \\
\hline B1.2.2 & 63.95619 & -16.84228 & 0.46 & $1000 * 650$ & 0.8 & 44.00 \\
\hline B2.1.1 & 63.95761 & -16.84492 & 0.4 & $850 * 500$ & 0.63 & 36.43 \\
\hline B2.1.2 & 63.95761 & -16.84492 & 0.4 & $850 * 500$ & 0.63 & 36.43 \\
\hline B2.2.1 & 63.95761 & -16.84503 & 0.48 & $870 * 660$ & 1.05 & 54.54 \\
\hline B2.2.2 & 63.95761 & -16.84503 & 0.48 & $870 * 660$ & 0.63 & 36.43 \\
\hline B3.1.1 & 63.95867 & -16.84628 & 0.3 & $960 * 500$ & 0.32 & 21.33 \\
\hline B3.1.2 & 63.95867 & -16.84628 & 0.3 & $960 * 500$ & 0.29 & 19.74 \\
\hline C1.1 & 63.95419 & -16.84831 & 0.45 & $600 * 450$ & 0.7 & 39.59 \\
\hline C1.2 & 63.95419 & -16.84831 & 0.45 & $600 * 450$ & 0.67 & 38.25 \\
\hline $\mathrm{C} 2.1$ & 63.95500 & -16.84858 & 0.45 & & 0.65 & 37.34 \\
\hline $\mathrm{C} 2.2$ & 63.95500 & -16.84858 & 0.45 & & 0.65 & 37.34 \\
\hline C 3.1 & 63.95803 & -16.85008 & 0.45 & $500 * 300$ & 0.35 & 22.90 \\
\hline D1.1 & 63.94631 & -16.85231 & 0.45 & $700 * 500$ & 0.6 & 35.05 \\
\hline D1.2 & 63.94631 & -16.85231 & 0.45 & $700 * 500$ & 0.6 & 35.05 \\
\hline $\mathrm{D} 2.1$ & 63.94758 & -16.85486 & 0.45 & $750 * 500$ & 0.58 & 34.13 \\
\hline $\mathrm{D} 2.2$ & 63.94758 & -16.85486 & 0.45 & $750 * 500$ & 0.57 & 33.66 \\
\hline D3.1 & 63.94869 & -16.85711 & 0.45 & $700 * 650$ & 0.7 & 39.59 \\
\hline D3.2 & 63.94869 & -16.85711 & 0.45 & $700 * 650$ & 0.6 & 35.05 \\
\hline E1.1 & 63.94253 & -16.85747 & 0.45 & $770 * 660$ & 0.7 & 39.59 \\
\hline E1.2 & 63.94253 & -16.85747 & 0.45 & $770 * 660$ & 0.7 & 39.59 \\
\hline E2.1 & 63.94383 & -16.85808 & 0.47 & $600 * 500$ & 0.4 & 25.45 \\
\hline E2.2 & 63.94383 & -16.85808 & 0.47 & $600 * 500$ & 0.4 & 25.45 \\
\hline E3.1 & 63.94664 & -16.86064 & 0.48 & $670 * 550$ & 0.4 & 25.45 \\
\hline E3.2 & 63.94664 & -16.86064 & 0.48 & $670 * 550$ & 0.4 & 25.45 \\
\hline F1.1 & 63.93950 & -16.86256 & 0.44 & $640 * 620$ & 0.5 & 30.35 \\
\hline
\end{tabular}




\begin{tabular}{|c|c|c|c|c|c|c|}
\hline Test ID & Northing & Easting & $\begin{array}{l}\text { Hole depth } \\
\text { (m) }\end{array}$ & $\begin{array}{ll}\text { Hole } & \text { size } \\
(\mathrm{mm}) & \end{array}$ & d10 & $K \mathbf{~ m} / \mathbf{d}$ \\
\hline $\bar{F} 1.2$ & 63.93950 & -16.86256 & 0.44 & $640 * 620$ & 0.65 & 37.34 \\
\hline F2.1 & 63.94044 & -16.86561 & 0.52 & $610 * 490$ & 0.55 & 32.73 \\
\hline F2.2 & 63.94044 & -16.86561 & 0.52 & $610 * 490$ & 0.55 & 32.73 \\
\hline F3.1 & 63.94142 & -16.86928 & 0.43 & $560 * 410$ & 0.05 & 4.92 \\
\hline F3.2 & 63.94142 & -16.86928 & 0.43 & $560 * 410$ & 0.65 & 37.34 \\
\hline F4.1 & 63.94197 & -16.87069 & 0.4 & $440 * 360$ & 0.25 & 17.55 \\
\hline $\mathrm{F} 4.2$ & 63.94197 & -16.87069 & 0.4 & $440 * 360$ & 0.25 & 17.55 \\
\hline
\end{tabular}

MacDonald, A. M., Maurice, L., Dobbs, M. R., Reeves, H. J., and Auton, C. A.: Relating in situ hydraulic conductivity, particle size and relative density of superficial deposits in a heterogeneous catchment. J. Hydrol., 434-435, 130-141, https://doi.org/10.1016/j.jhydrol.2012.01.018, 2012. 

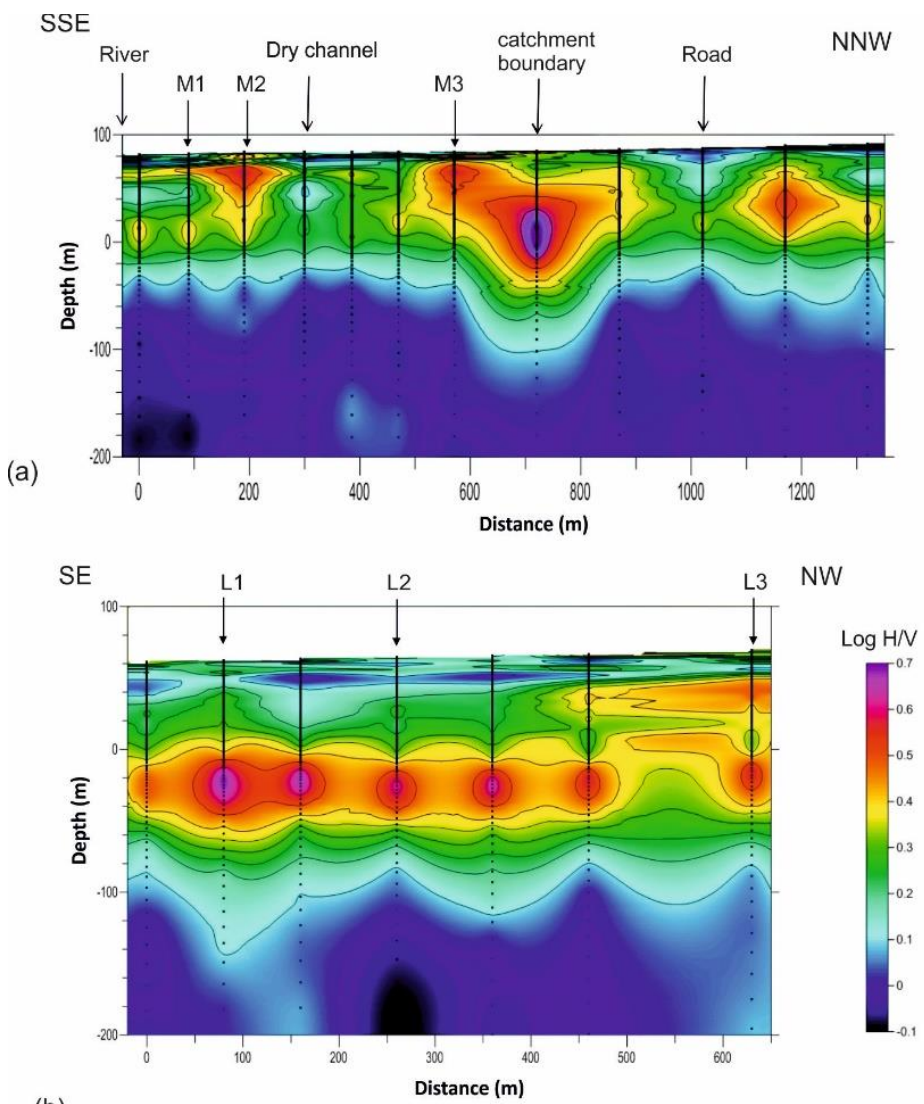

(b)

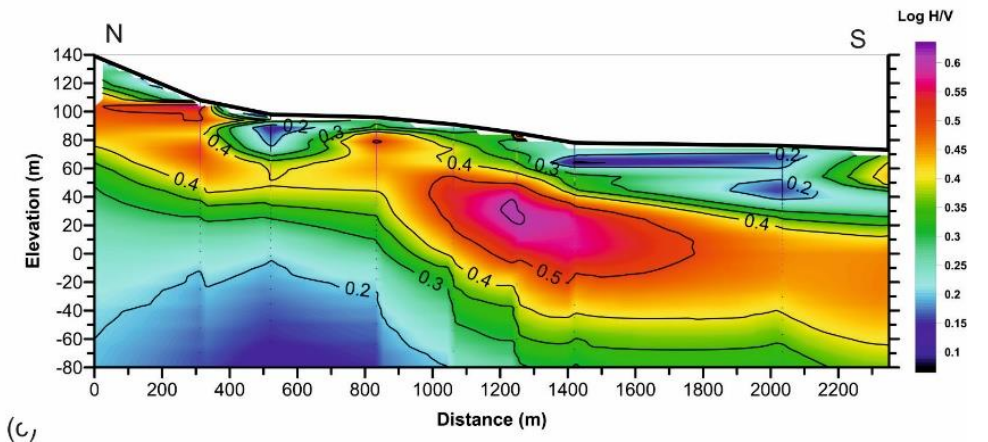

Figure S1. Log H/V from Tromino passive seismic surveys (see Methods). (a) Upper Sandur (b) Lower Sandur and (c) transect away from the glacier margin at the neighbouring sandur at Svinafellsjokul 
Table S4 Summary of selected chemical parameters, temperature and stable isotopes of river water and of groundwater in sandur aquifer in individual piezometers (piezometer locations in Fig. 1) and all springs grouped together. Data from six sampling campaigns (three summer and three winter); annual means calculated by weighting seasonal means to season length (summer $=\mathbf{0 . 4 2}$; winter $=\mathbf{0 . 5 8}$ ), except temperature data for piezometer groundwater: data derived from continuous monitoring in piezometers at 15 minute intervals for 34 months, at 7-8.4 m depth; annual mean unweighted. sd - standard deviation of whole dataset; $\mathbf{n}$ - number of samples. The full dataset is available (MacDonald et al., 2019)

\begin{tabular}{|c|c|c|c|c|c|c|c|c|c|c|c|c|c|c|c|}
\hline & \multicolumn{3}{|c|}{ SEC $\mu \mathrm{S} \mathrm{cm}^{-1}$} & \multicolumn{2}{|c|}{$\mathrm{HCO}_{3} \mathrm{mg} \mathrm{l}^{-1}$} & \multicolumn{3}{|c|}{ Temperature $^{\circ} \mathrm{C}$} & \multicolumn{3}{|c|}{$\delta^{18} \mathrm{O} \%$} & \multicolumn{3}{|c|}{ б2H\%o } & \multirow[b]{2}{*}{ Mean } \\
\hline & Mean $^{1}$ & $\mathrm{sd}$ & $\mathrm{n}$ & Mean $^{1}$ & $\mathrm{sd}$ & $\mathrm{n}$ & Mean $^{2}$ & $\mathrm{sd}$ & $\mathrm{n}$ & Mean $^{1}$ & $\mathrm{sd}$ & $\mathrm{n}$ & Mean $^{1}$ & $\mathrm{sd}$ & \\
\hline U1 & 62.58 & 14.10 & 6 & 28.77 & 6.23 & 5 & 2.35 & 0.75 & 115274 & -10.2 & 0.2 & 6 & -72.0 & 2.1 & 6 \\
\hline $\mathrm{U} 2$ & 98.85 & 10.53 & 5 & 44.42 & 1.03 & 4 & 4.94 & 0.10 & 115169 & -8.0 & 0.04 & 5 & -59.1 & 0.7 & 5 \\
\hline M1 & 69.59 & 8.91 & 5 & 31.49 & 1.71 & 4 & 5.01 & 1.30 & 115209 & -9.4 & 0.3 & 5 & -67.4 & 2.6 & 5 \\
\hline M2 & 78.37 & 13.04 & 5 & 36.51 & 3.13 & 4 & 5.26 & 1.06 & 115095 & -8.7 & 0.4 & 5 & -63.5 & 3.9 & 5 \\
\hline M3 & 78.21 & 13.99 & 5 & 37.03 & 4.02 & 4 & 4.69 & 1.06 & 114905 & -8.3 & 0.6 & 5 & -60.0 & 4.2 & 5 \\
\hline L1 & 62.75 & 6.21 & 5 & 27.33 & 2.72 & 4 & 3.39 & 0.98 & 114841 & -10.6 & 0.1 & 5 & -74.2 & 1.0 & 5 \\
\hline L2 & 55.39 & 6.10 & 5 & 25.59 & 0.3 & 4 & 3.19 & 1.39 & 114866 & -10.7 & 0.4 & 5 & -75.2 & 3.4 & 5 \\
\hline L3 & 96.14 & 6.98 & 5 & 47.68 & 3.58 & 4 & 4.65 & 0.17 & 114671 & -8.9 & 0.2 & 5 & -63.5 & 1.3 & 5 \\
\hline Springs & 68.18 & 21.34 & 23 & 32.64 & 7.16 & 4 & 6.05 & 3.55 & 21 & -9.1 & 1.0 & 23 & -64.8 & 7.0 & 23 \\
\hline River & 43.00 & 10.51 & 19 & 20.66 & 5.43 & 9 & 1.69 & 0.65 & 16 & -10.9 & 0.4 & 19 & -76.1 & 2.6 & 19 \\
\hline
\end{tabular}

${ }^{1}$ Weighted annual mean

${ }^{2}$ Weighted annual mean for springs and river; unweighted for piezometers

MacDonald, A. M., Ó Dochartaigh, B. É., Fallas, H.: Water chemistry and stable isotope data, Virkisjokull Glacier Observatory, 2011-2018. British Geological Survey.

(Dataset). https://dx.doi.org/10.5285/14da9c02-c5ec-4019-8e5c-06c744d8be9d, 2019. 